\title{
Associação de deltametrina com Acarophenax lacunatus e seu impacto sobre o desenvolvimento de Rhyzopertha dominica
}

\author{
José Roberto Gonçalves ${ }^{(1)}$, Lêda Rita D'Antonino Faroni(2), Raul Narciso Carvalho Guedes ${ }^{(1)}$, \\ Carlos Romero Ferreira de Oliveira ${ }^{(1)}$ e Flávia Miranda Garcia(2)
}

\begin{abstract}
(1)Universidade Federal de Viçosa (UFV), Dep. de Biologia Animal, Av. P.H. Rolfs s/non, CEP 36570-000, Viçosa, MG. E-mail: goncalves_mip@hotmail.com, guedes@ufv.br, crfoliveira@hotmail.com (2)UFV, Dep. de Engenharia Agrícola. E-mail: Ifaroni@ufv.br, fgarcia_agroufv@hotmail.com
\end{abstract}

\begin{abstract}
Resumo - O objetivo deste trabalho foi avaliar a associação do inseticida deltametrina, aplicado em grãos de trigo, com o ácaro parasita Acarophenax lacunatus e seu impacto sobre o desenvolvimento de Rhyzopertha dominica. Grãos de trigo ( $13 \%$ de teor de água) foram tratados com diferentes doses de deltametrina $(0,00,0,125$, $0,25,0,375,0,50,0,625,0,75,0,875$ e $1,00 \mathrm{mg}^{\text {i.a. }} \mathrm{kg}^{-1}$ ). As unidades experimentais consistiram de placas de Petri contendo $30 \mathrm{~g}$ de grãos tratados, ou não, com o inseticida, infestados com 30 adultos de $R$. dominica. Cinco dias depois da infestação, foram inoculados cinco ácaros parasitas por unidade experimental, em sete repetições. As unidades experimentais foram armazenadas por 60 dias depois da infestação em câmara climatizada ajustada a $30 \pm 1^{\circ} \mathrm{C}, 60 \pm 5 \%$ UR e escotofase de 24 horas. A taxa instantânea de crescimento de $R$. dominica apresentou índices negativos para as doses de deltametrina maiores que $0,25 \mathrm{mg}$ i.a. $\mathrm{kg}^{-1}$. A. lacunatus associado a doses de deltametrina menores que $0,5 \mathrm{mg}$ i.a. $\mathrm{kg}^{-1}$ reduz as fases imaturas de $R$. dominica.
\end{abstract}

Termos para indexação: Acarophenacidae, piretróide, manejo integrado de pragas.

\section{Association of deltamethrin with Acarophenax lacunatus on development of Rhyzopertha dominica}

\begin{abstract}
The objective of this work was to evaluate the development of Rhyzopertha dominica on wheat grain treated with the insecticide deltamethrin and infested with the mite Acarophenax lacunatus. The experimental units were Petri dishes containing $30 \mathrm{~g}$ of wheat grain (13\% moisture content) sprayed with different doses of deltamethrin $\left(0.00,0.125,0.25,0.375,0.50,0.625,0.75,0.875\right.$ and $1.00 \mathrm{mg}$ a.i. $\left.\mathrm{kg}^{-1}\right)$ infested with 30 adults of $R$. dominica. Five days after the infestation, five mites were inoculated on each experimental unit, in seven replicates. All treatments were maintained under controlled conditions $\left(30 \pm 1^{\circ} \mathrm{C}, 60 \pm 5 \%\right.$ r.h. and 24 hours scotophase) for 60 days after the infestation. The instantaneous rate of increase of $R$. dominica was negative under doses higher than $0.25 \mathrm{mg}$ a.i. $\mathrm{kg}^{-1}$. The lowest number of immature $R$. dominica was observed with the interaction between deltamethrin doses smaller than $0.5 \mathrm{mg}$ a.i. $\mathrm{kg}^{-1}$ and $A$. lacunatus.
\end{abstract}

Index terms: Acarophenacidae, pyrethroid, integrated pest management.

\section{Introdução}

O coleóptero Rhyzopertha dominica (Fabricius) (Bostrichidae) é a principal praga de trigo armazenado no Brasil (Lorini \& Galley, 2000). A forma mais utilizada de prevenção e controle desse inseto nas unidades armazenadoras é por meio de inseticidas, em virtude da facilidade de aplicação e da maior rapidez de ação (White \& Leesch, 1996; Mourier \& Poulsen, 2000). Um dos inseticidas com maior proteção dos grãos é o piretróide deltametrina (Guedes, 1990; Beckel et al., 2004).
A resistência de $R$. dominica a produtos químicos deve ser considerada no processo de armazenamento de grãos, uma vez que pode inviabilizar o uso de inseticidas disponíveis no mercado e causar perdas dos investimentos financeiros (White \& Leesch, 1996). Por essa razão, o uso de inimigos naturais resistentes ou tolerantes a pesticidas tem sido um instrumento na supressão de populações dessa praga, que persiste em ambientes de armazenamento, depois do tratamento químico (Gonçalves et al., 2002). 
O ácaro parasita Acarophenax lacunatus (Cross \& Krantz) (Acarophenacidae) pode ser uma alternativa promissora ao manejo de populações de $R$. dominica resistentes a inseticidas, uma vez que esse inimigo natural persistiu parasitando ovos desse coleóptero em grãos de trigo tratados com os inseticidas deltametrina, bifentrina, pirimifós metílico e fenitrotion (Gonçalves et al., 2004). Inimigos naturais que parasitam ovos conseguem matar a praga antes que ela venha causar dano (Hansen \& Jensen, 2002), e A. lacunatus apresenta essa vantagem (Faroni et al., 2000). Isto é importante devido ao fato de apenas uma fêmea de $R$. dominica ser capaz de ovipositar uma média de 420 ovos em condições de temperatura ótima $\left(32^{\circ} \mathrm{C}\right)$, podendo aumentar consideravelmente sua população em um curto intervalo de tempo (Faroni \& García-Mari, 1992), se métodos de controle não forem utilizados (King \& Nordlund, 1992).

Os inimigos naturais liberados nos armazéns podem se reproduzir por muito tempo, quando possuem hospedeiros disponíveis e condições ambientais satisfatórias (Schöller et al., 1997). Ao mesmo tempo, as estruturas dos armazéns (silos, graneleiros etc.) proporcionam ambiente adequado para o desenvolvimento desses organismos, o que tem despertado o interesse de pesquisadores em estudar agentes de controle biológico que possam ser utilizados como instrumento do manejo integrado de pragas de grãos armazenados (Haines, 1984; Brower, 1990; Schöller et al., 1997; Gonçalves et al., 2002).

Este trabalho teve como objetivo avaliar a influência da associação de deltametrina aplicado em grãos de trigo e do ácaro parasita A. lacunatus sobre o desenvolvimento de $R$. dominica.

\section{Material e Métodos}

A espécie $R$. dominica foi criada em câmara climatizada, à temperatura de $30 \pm 1^{\circ} \mathrm{C}, 60 \pm 5 \%$ UR e escotofase de 24 horas, a partir de adultos provenientes da criação estoque do laboratório, iniciada com 50 adultos de $R$. dominica em placas de Petri contendo grãos de trigo, previamente expurgados, com teor de água de $13 \%$ b.u. (base úmida). Os ovos foram coletados com peneira de orifícios de $1 \mathrm{~mm}$ de diâmetro, sete dias depois da montagem das criações, tempo suficiente para que as fêmeas iniciassem a oviposição, e colocados em outras placas com a mesma dieta. Esses ovos foram coletados de acordo com um calendário, para que houves- se disponibilidade de adultos de $R$. dominica com idade conhecida. O controle da infestação de A. lacunatus sobre as criações de $R$. dominica foi realizado por meio da utilização de 0,6 mg de enxofre por grama de grãos de trigo (Gonçalves, 2005).

Indivíduos de $A$. lacunatus foram obtidos de criações massais de $R$. dominica infestadas por esse parasita há mais de quatro anos. Esse ácaro foi criado em populações de $R$. dominica a $30 \pm 1^{\circ} \mathrm{C}, 60 \pm 5 \%$ UR e escotofase de 24 horas.

No ensaio, foram utilizados grãos de trigo com 13\% de teor de água tratados com as seguintes doses de deltametrina (K-Obiol $25 \mathrm{CE}$ ): 0,00, 0,125, 0,25, 0,375, $0,50,0,625,0,75,0,875$ e $1,00 \mathrm{mg}$ i.a. $\mathrm{kg}^{-1}$, as quais foram dissolvidas em água destilada, separadamente, e pulverizadas com atomizador manual, com o bico voltado para baixo, a $20 \mathrm{~cm}$ de altura da massa de 1,0 kg de grãos de trigo, em local protegido do vento. $\mathrm{O}$ volume de solução aplicado foi de $2 \mathrm{~mL}$ por massa de grãos, correspondendo a $2 \mathrm{~L}$ de solução por $1.000 \mathrm{~kg}$ de grãos. $\mathrm{O}$ atomizador foi calibrado em teste preliminar, em uma superfície de $1,0 \mathrm{~m}^{2}$ de área, com $10 \mathrm{~kg}$ de grãos de trigo, ajustando-se o volume de $2 \mathrm{~mL}$ de solução para cada quilograma de grãos de trigo. Quanto à testemunha, utilizou-se somente água destilada na pulverização. Em seguida, aguardou-se um período de 30 minutos para a infestação de $R$. dominica (Gonçalves et al., 2002).

As unidades experimentais consistiram de placas de Petri de 140x10 mm (diâmetro x altura), contendo $30 \mathrm{~g}$ de grãos, infestados com 30 adultos de $R$. dominica nãosexados, com idade entre três e sete dias, com inoculação e sem inoculação de A. lacunatus. Cinco dias depois da infestação, período suficiente para que esse coleóptero colocasse os primeiros ovos, parte das unidades experimentais foram inoculadas com cinco fêmeas adultas de A. lacunatus, em sete repetições. As placas foram revestidas com filme de PVC para evitar que os insetos e os ácaros escapassem e também para prevenir contaminação. Em cada placa, foram feitos três furos com alfinete entomológico no filme de PVC para permitir melhor troca de ar com o meio externo. Todas as unidades experimentais foram armazenadas por 60 dias em câmara climatizada ajustada a $30 \pm 1^{\circ} \mathrm{C}$, $60 \pm 5 \%$ UR e escotofase de 24 horas.

Depois do período de armazenamento, avaliou-se o efeito da associação de deltametrina com o ácaro A. lacunatus sobre o desenvolvimento de $R$. dominica. Para isso, a massa de grãos de cada placa foi passada 
por peneira, com orifícios de 1,0 mm de diâmetro, separando-se os adultos de $R$. dominica dos grãos e o resíduo (pó), contendo fases imaturas (ovos e larvas) desse coleóptero e adultos de A. lacunatus. Em seguida, procedeu-se à determinação da perda de massa dos grãos de trigo (\%), por meio da alteração da massa com o término do experimento, e à contagem do número de adultos vivos de $R$. dominica. O resíduo da massa de grãos foi analisado, com auxílio de um microscópio estereoscópico, para determinação do número de ovos de $R$. dominica, parasitados ou não por A. lacunatus, do número de fêmeas desse ácaro, em processo de fisogastria, e do número de larvas de primeiro ínstar de R. dominica.

A taxa instantânea de crescimento de $R$. dominica $\left(r_{i}\right)$ foi calculada por meio da equação:

$\mathrm{r}_{\mathrm{i}}=\left[\ln \left(\mathrm{N}_{\mathrm{f}} / \mathrm{N}_{0}\right)\right] / \Delta \mathrm{t}$; em que $\mathrm{N}_{\mathrm{f}}$ é o número final de insetos vivos; $\mathrm{N}_{0}$ é o número inicial de insetos vivos; $\Delta \mathrm{t}$ é a variação de tempo (número de dias em que o ensaio foi executado) (Walthall \& Stark, 1997).

A fim de avaliar a associação de deltametrina com A. lacunatus e seu impacto sobre o desenvolvimento de $R$. dominica foram realizadas análises de regressão. A perda de massa dos grãos de trigo e a taxa instantânea de crescimento de $R$. dominica foram comparadas por análise de correlação de Pearson.

\section{Resultados e Discussão}

A taxa instantânea de crescimento populacional $\left(\mathrm{r}_{\mathrm{i}}\right)$ de $R$. dominica apresentou diferença significativa quanto às doses de deltametrina $\left(\mathrm{F}_{8 ; 97}=15,67 ; \mathrm{p}<0,001\right) \mathrm{e}$ correlação positiva para perda de massa dos grãos de trigo $(r=0,78 ; p=0,0001)$. Os tratamentos com doses de deltametrina maiores que $0,375 \mathrm{mg}$ i.a. $\mathrm{kg}^{-1}$ proporcionaram as menores perdas de massa dos grãos (menos de 4\%), comparadas com a testemunha que teve uma perda de aproximadamente $50 \%$. Entretanto, a interação das doses de deltametrina com $A$. lacunatus não apresentou diferença significativa quanto a esses parâmetros, demonstrando que apenas o inseticida proporcionou maior proteção dos grãos e menor desenvolvimento desse coleóptero. Isto foi mais evidente nas doses de deltametrina acima de 0,25 mg i.a. $\mathrm{kg}^{-1}$, em que foram observadas taxas instantâneas de crescimento populacional negativa para R. dominica (Figura 1).

Embora tenha ocorrido sobrevivência de $R$. dominica nas doses de deltametrina superiores a $0,25 \mathrm{mg}$ i.a. $\mathrm{kg}^{-1}$, não houve crescimento da população desse inseto-praga.
Em períodos de armazenamento maiores que 60 dias, essa situação poderia ser modificada, com possível crescimento populacional desse inseto-praga, principalmente, por se tratar de uma espécie com elevada capacidade de multiplicação e resistência a deltametrina (Faroni \& García-Mari, 1992; Lorini \& Galley, 2000). Desta forma, as unidades armazenadoras de grãos devem ser monitoradas freqüentemente, uma vez que existe a possibilidade de esse inseto-praga se desenvolver na massa de grãos, mesmo quando tiver sido tratada com doses elevadas de um inseticida protetor (Hagstrum \& Subramanyam, 2000).

A resistência de linhagens de $R$. dominica a inseticidas tem contribuído para o aumento das doses dos ingredientes ativos, nas unidades armazenadoras, na busca de controle mais efetivo das pragas, o que nem sempre atinge resultados satisfatórios (Beckel et al., 2004). Este fato pode ser observado nas doses de deltametrina acima de $0,75 \mathrm{mg}$ i.a. $\mathrm{kg}^{-1}$, as quais proporcionaram reduções semelhantes da taxa instantânea de crescimento populacional de R. dominica (Figura 1). Nesta situação, não é aconselhado aumentar a dose do inseticida, mas tentar utilizar outras formas de controle associadas ao método químico (King \& Nordlund, 1992; Hagstrum \& Subramanyam, 2000).

Apesar de o aumento da dose de deltametrina ter favorecido maior proteção dos grãos de trigo, este tipo de manejo de $R$. dominica pode acarretar resistência desse inseto-praga ao ingrediente ativo, além de causar problemas de resíduos tóxicos nos produtos

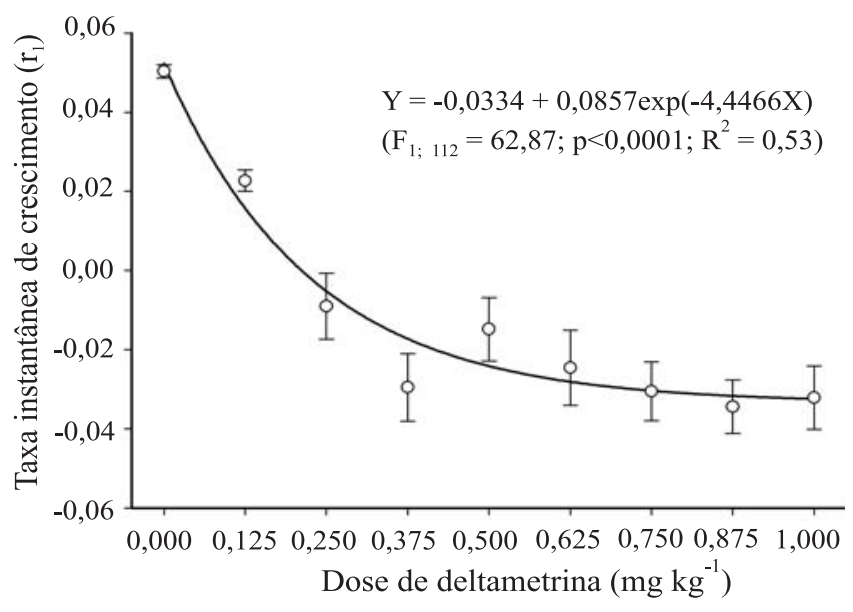

Figura 1. Desenvolvimento de uma população de Rhyzopertha dominica depois de 60 dias do armazenamento de grãos de trigo, tratados com diferentes doses de deltametrina. 
processados (Beckel et al., 2004). Uma alternativa para amenizar esses entraves pode ser feita com a associação de inimigos naturais e doses de inseticidas, que sejam eficazes contra as pragas e seletivos em relação aos organismos benéficos (Gonçalves et al., 2002). Isto foi observado nas doses de deltametrina entre 0,375 e $0,875 \mathrm{mg}$ i.a. $\mathrm{kg}^{-1}$, que, além de permitir o desenvolvimento do parasita A. lacunatus, apresentaram bons resultados no controle dos imaturos de $R$. dominica (Figuras 2 e 3). De acordo com Hagstrum \& Subramanyam (2000), esse manejo, além de favorecer a sobrevivência dos inimigos naturais, contribui com o uso racional dos inseticidas.

Na ausência do ácaro parasita, tanto o número de ovos $\left(\mathrm{F}_{8 ; 108}=2,17 ; \mathrm{p}=0,03\right)$ quanto o de larvas
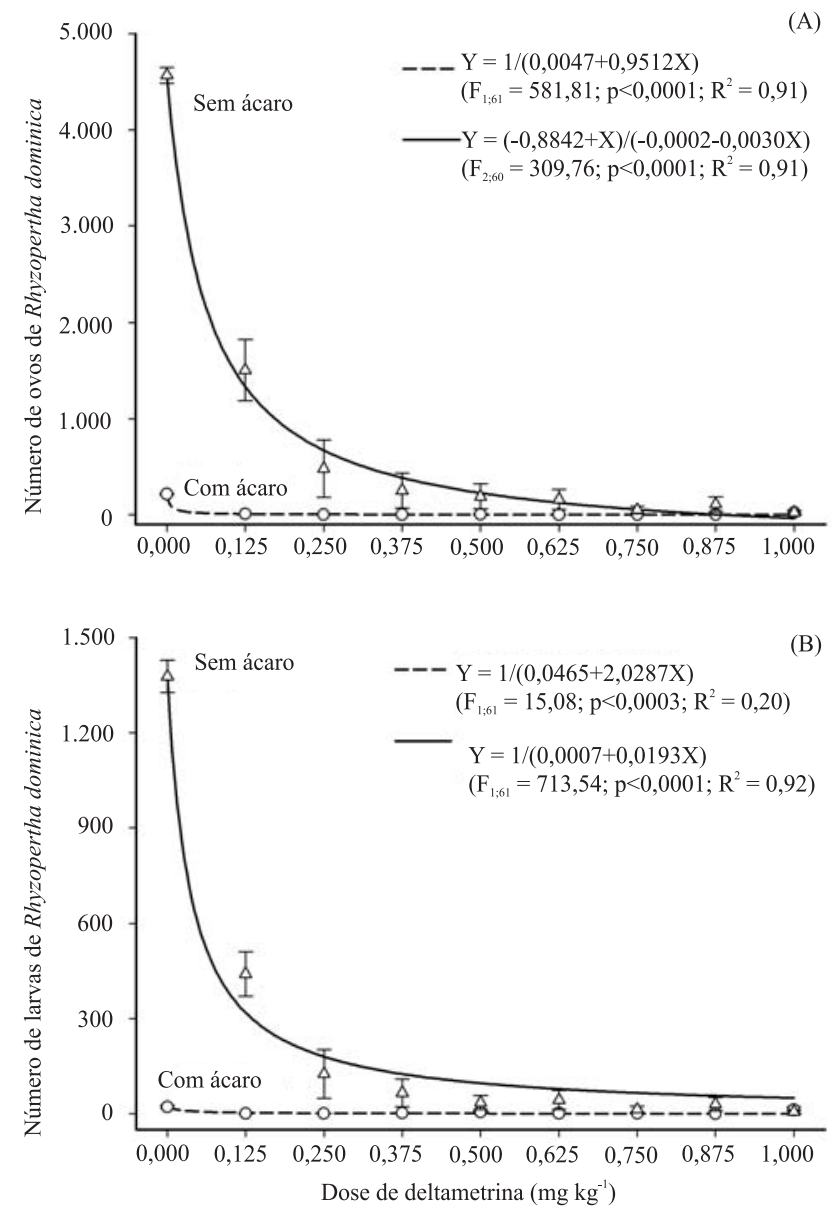

Figura 2. Número de ovos não-parasitados (A) e larvas (B) de uma população de Rhyzopertha dominica sobre grãos de trigo tratados com diferentes doses de deltametrina associadas ao parasita Acarophenax lacunatus, depois de 60 dias de armazenamento.
$\left(\mathrm{F}_{8 ; 108}=5,08 ; \mathrm{P}<0,001\right)$ de $R$. dominica demonstraram tendência decrescente com o aumento das doses do inseticida. Tal tendência também foi observada na presença de $A$. lacunatus, quando o número de ovos e larvas desse coleóptero apresentou redução acentuada em todas as doses de deltametrina. Porém, com o aumento progressivo das doses desse inseticida, constatou-se que a presença ou não do ácaro deixa de influenciar significativamente o número de ovos e larvas de $R$. dominica (Figura 2). Por essa razão, a associação de deltametrina com A. lacunatus não apresentou, no período estudado, supressão populacional significativa de adultos de $R$. dominica. Por sua vez, em doses menores que $0,5 \mathrm{mg}$ i.a. $\mathrm{kg}^{-1}$, essa interação foi eficaz sobre as fases imaturas desse inseto-praga. Esse fato é importante, uma vez que uma fêmea de $R$. dominica em condições climáticas favoráveis é capaz de ovipositar grande número de ovos, ocorrendo, em pouco tempo, o crescimento da população desse inseto-praga (Faroni \& García-Mari, 1992).

A eficácia da associação do controle químico com inimigos naturais pode ser melhorada por meio da interação com o controle físico (aeração), além de medidas de limpeza e higienização das unidades armazenadoras, uma vez que esses pré-requisitos são fundamentais para reduzir o desenvolvimento de pragas nesses ambientes. Esses procedimentos têm sido considerados primordiais para associação do controle biológico, uma vez que muitos inimigos naturais são mais eficazes em baixas populações das pragas (Smith, 1994; Zdarkova, 1996; Flinn, 1998).

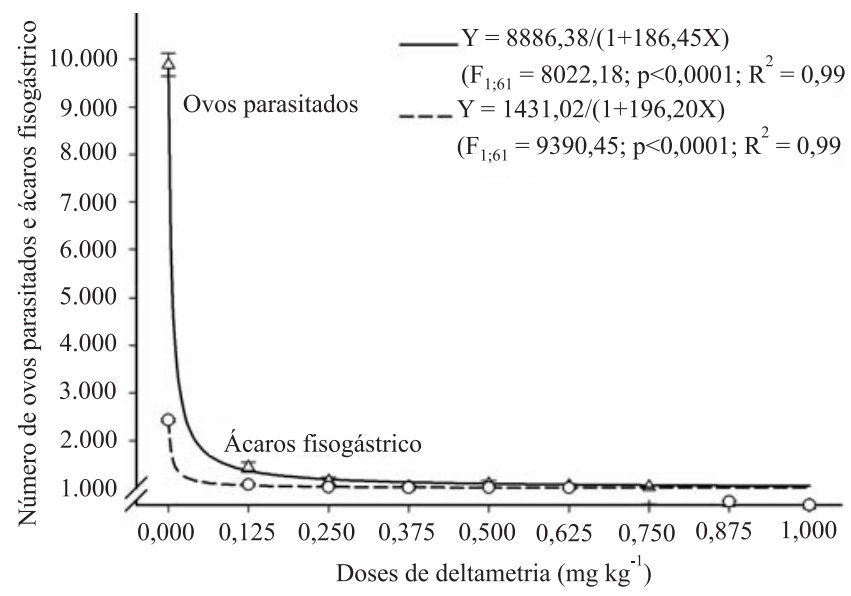

Figura 3. Número de ovos de Rhyzopertha dominica parasitados e de fêmeas fisogástricas de Acarophenax lacunatus, 60 dias depois do armazenamento de grãos de trigo, tratados com diferentes doses de deltametrina, infestados com $R$. dominica. 
O número de ovos de $R$. dominica parasitados $\left(\mathrm{F}_{8 ; 54}=24,02 ; \mathrm{p}<0,001\right)$ e o de fêmeas fisogástricas de A. lacunatus $\left(\mathrm{F}_{8 ; 54}=51,88 ; \mathrm{p}<0,001\right)$ apresentaram diferença significativa em relação a doses de deltametrina. Embora em menor magnitude do que a testemunha, A. lacunatus parasitou ovos de $R$. dominica em doses crescentes de deltametrina até $0,875 \mathrm{mg}$ i.a. $\mathrm{kg}^{-1}$ (Figura 3). No entanto, uma das restrições ao uso de inimigos naturais em produtos armazenados é devido aos fragmentos que estes podem deixar no produto beneficiado (farinha), mas, por se tratar de espécies pequenas, esses organismos são facilmente removidos no processo de limpeza, antes do beneficiamento dos grãos (Haines, 1984; Brower, 1990; Schöller et al., 1997). Flinn \& Hagstrum (2001) relatam que o uso do parasitóide Theocolax elegans (Westwood) (Pteromalidae), além de reduzir danos causados por $R$. dominica, também reduziu os fragmentos desses insetos na farinha. Isso demonstra a possibilidade de utilizar o controle biológico como prática auxiliar para o controle de pragas em armazenamento.

\section{Conclusões}

1. A associação de deltametrina com Acarophenax lacunatus não proporciona redução da taxa instantânea de crescimento de Rhyzopertha dominica num período de 60 dias do armazenamento dos grãos de trigo.

2. Doses de deltametrina maiores que $0,375 \mathrm{mg}$ i.a. $\mathrm{kg}^{-1}$ proporcionam menor desenvolvimento de $R$. dominica e maior proteção dos grãos.

3. A. lacunatus reduz as fases imaturas de $R$. dominica, quando associado a doses de deltametrina menores que $0,5 \mathrm{mg}$ i.a. $\mathrm{kg}^{-1}$.

\section{Agradecimentos}

À Capes, pelo apoio financeiro; à Universidade Federal de Viçosa, pela oportunidade de realizar o trabalho.

\section{Referências}

BECKEL, H.; LORINI, I.; LÁZZARI, S.M.N. Comportamento de adultos de diferentes raças de Rhyzopertha dominica (Fabricius) (Coleoptera, Bostrichidae) em superfície tratada com deltamethrin. Revista Brasileira de Entomologia, v.48, p.115-118, 2004.

BROWER, J.H. Pests of stored products. In: HABECK, D.H.; BENNETT, F.D.; FRANK, J.H. (Ed.). Classical biological control in the southern United States. Gainesville: IFAS Editorial, University of Florida, 1990. p.113-122.
FARONI, L.R.A.; GARCÍA-MARI, F. Influencia de la temperatura sobre los parámetros biológicos de Rhyzopertha dominica (F.). Boletín de Sanidad Vegetal, v.18, p.455-467, 1992.

FARONI, L.R.A.; GUEDES, R.N.C.; MATIOLI, A.L. Potential of Acarophenax lacunatus (Prostigmata: Acarophenacidae) as a biological control agent of Rhyzopertha dominica (Coleoptera: Bostrichidae). Journal of Stored Products Research, v.36, p.5563, 2000.

FLINN, P.W. Temperature effects on efficacy of Choetospila elegans (Hymenoptera: Pteromalidae) to suppress Rhyzopertha dominica (Coleoptera: Bostrichidae) in stored wheat. Journal of Economic Entomology, v.91, p.320-323, 1998.

FLINN, P.W.; HAGSTRUM, D.W. Augmentative releases of parasitoid wasps in stored wheat reduces insect fragments in flour. Journal of Stored Products Research, v.37, p.179-186, 2001.

GONÇALVES, J.R. Interações de Acarophenax lacunatus com deltametrina, temperatura e o parasitóide Anisopteromalus calandrae na supressão populacional de Rhyzopertha dominica. 2005. 91p. Tese (Doutorado) - Universidade Federal de Viçosa, Viçosa.

GONÇALVES, J.R.; FARONI, L.R.A.; GUEDES, R.N.C. Pyrethoid-Acarophenax lacunatus interaction in suppressing the beetle Rhyzopertha dominica on stored wheat. Experimental and Applied Acarology, v.26, p.231-242, 2002.

GONÇALVES, J.R.; FARONI, L.R.A.; GUEDES, R.N.C.; OLIVEIRA, C.R.F. Insecticide selectivity to the parasitic mite Acarophenax lacunatus (Cross \& Krantz) (Prostigmata: Acarophenacidae) on Rhyzopertha dominica (Fabr.) (Coleoptera: Bostrichidae). Neotropical Entomology, v.33, p.243-248, 2004.

GUEDES, R.N.C. Manejo integrado para a proteção de grãos armazenados contra insetos. Revista Brasileira de Armazenamento, v.15, p.3-48, 1990.

HAGSTRUM, D.W.; SUBRAMANYAM, B. Monitoring and decision tools. In: SUBRAMANYAM, B.; HAGSTRUM, D.W. (Ed.). Alternatives to pesticides in stored-product IPM. London: Kluwer Academic Publishers, 2000. p.1-28.

HAINES, C.P. Biological methods for integrated control of insects and mites in tropical stored products. III. The use of predators and parasites. Tropical Stored Products Information, v.48, p.17-25, 1984.

HANSEN, L.S.; JENSEN, K.M.V. Effect of temperature on parasitism and host-feeding of Trichogramma turkestanica (Hymenoptera: Trichogrammatidae) on Ephestia kuehniella (Lepidoptera: Pyralidae). Journal of Economic Entomology, v.95, p.50-56, 2002.

KING, E.G.; NORDLUND, D.A. Propagation and augmentative releases of predators and parasitoids for control of arthropod pests. Pesquisa Agropecuária Brasileira, v.27, p.239-254, 1992.

LORINI, I.; GALLEY, D.J. Estimation of realized heritability of resistance to deltamethrin insecticide in selected strains of Rhyzopertha dominica (F.) (Coleoptera: Bostrychidae). Journal of Stored Products Research, v.36, p.119-124, 2000.

MOURIER, H.; POULSEN, K.P. Control of insects and mites in grain using a high temperature/short time (HTST) technique. Journal of Stored Products Research, v.6, p.309-318, 2000. 
SCHÖLLER, M.; PROZELL, S.; AL-KIRSHI, A.G.; REICHMUTH, C. Towards biological control as a major component of integrated pest management in stored product protection. Journal of Stored Products Research, v.33, p.81-97, 1997.

SMITH, L. Computer simulation model for biological control of maize weevil by the parasitoid Anisopteromalus calandrae. In: INTERNATIONAL WORKING CONFERENCE ON STOREDPRODUCT PROTECTION, 6., 1994, Wallingford. Proceedings. Wallingford: CAB International, 1994. p.1147-1151.

WALTHALL, W.K.; STARK, J.D. Comparison of two populationlevel ecotoxicological endpoints: the intrinsic $\left(r_{m}\right)$ and instantaneous $\left(r_{i}\right)$ rates of increase. Environmental Toxicology and Chemistry, v.16, p.1068-1073, 1997.

WHITE, N.D.G.; LEESCH, J.G. Chemical control. In: SUBRAMANYAM, B.; HAGSTRUM, D.W. (Ed.). Integrated management of insects in stored products. New York: Marcel Dekker, 1996. p.287-330.

ZDARKOVA, E. Control of stored food mites by non-chemical methods. In: INTERNATIONAL FORUM ON STORED PRODUCT PROTECTION AND POST-HARVEST TREATMENT OF PLANT PRODUCTS, 1996, Strasbourg. Proceedings. Strasbourg: Council Europe, 1996. p.165-169.

Recebido em 23 de agosto de 2005 e aprovado em 10 de maio de 2006 